\title{
Algebraic $p$-Adic $L$-Functions in Non-Commutative Iwasawa Theory
}

\author{
By
}

David BuRns*

\begin{abstract}
We define canonical algebraic $p$-adic $L$-functions in non-commutative Iwasawa theory and establish some of their basic properties.
\end{abstract}

\section{$\S 1 . \quad$ Introduction}

In this note we use a result of Schneider and Venjakob in [8] to define canonical 'algebraic $p$-adic $L$-functions' in non-commutative Iwasawa theory. This construction refines both the notion of 'characteristic element' introduced by Venjakob in [10] and the 'Akashi series' introduced by Coates, Schneider and Sujatha in [5] and also plays a key role in descent theory in non-commutative Iwasawa theory. Indeed, in joint work with Venjakob [3], the results proved here are used to establish a general descent theory that, for example, clarifies the precise connection between main conjectures of non-commutative Iwasawa theory in the spirit of Coates, Fukaya, Kato, Sujatha and Venjakob [4] and the relevant cases of the equivariant Tamagawa number conjecture of Flach and the present author [2].

The main contents of this note is as follows. In $\S 2$ we define a natural notion of algebraic $p$-adic $L$-function. In $\S 3$ we prove some of the basic functorial properties of these elements and show that they refine the 'Akashi series' introduced in [5]. In $\S 4$ we prove that algebraic $p$-adic $L$-functions are 'characteristic elements' in the sense of [10] (and [4]).

Communicated by A. Tamagawa. Received August 2, 2007. Revised February 6, 2008 . 2000 Mathematics Subject Classification(s): 11R23.

*King's College London, Department of Mathematics, London WC2R2LS, United Kingdom.

e-mail: david.burns@kcl.ac.uk

(c) 2009 Research Institute for Mathematical Sciences, Kyoto University. All rights reserved. 
It is a pleasure to thank Otmar Venjakob for interesting discussions and the referee for several useful remarks.

\section{$\S 2 . \quad$ Algebraic $p$-Adic $L$-Functions}

\section{§2.1. Preliminaries}

In the sequel 'module' means 'left module'. For any ring $R$ we write $D(R)$ for the derived category of $R$-modules. We also write $D^{\mathrm{fg}}(R)$, resp. $D^{\mathrm{p}}(R)$, for the full triangulated subcategory of $D(R)$ comprising complexes that are isomorphic to a bounded complex of finitely generated $R$-modules, resp. to an object of the category $C^{\mathrm{p}}(R)$ of bounded complexes of finitely generated projective $R$-modules.

We fix a prime $p$. For any $\mathbb{Z}_{p}$-module $M$ we write $M_{\text {tor }}$ for its torsion submodule and set $M_{\mathrm{tf}}:=M / M_{\mathrm{tor}}$. For any profinite group $J$ we write $\Lambda(J)$ for the 'Iwasawa algebra' $\varliminf_{U} \mathbb{Z}_{p}[J / U]$ where $U$ runs over all open normal subgroups of $J$ and the limit is taken with respect to the natural projection maps $\mathbb{Z}_{p}[J / U] \rightarrow \mathbb{Z}_{p}\left[J / U^{\prime}\right]$ for $U \subseteq U^{\prime}$. If $J$ is a compact $p$-adic Lie group, then $\Lambda(J)$ is a noetherian ring and we write $Q(J)$ for its total quotient ring.

\section{§2.2. Canonical Ore sets}

We assume to be given a compact $p$-adic Lie group $G$ and a normal subgroup $H$ of $G$ with the property that the quotient group $\Gamma:=G / H$ is isomorphic (topologically) to the additive group of $\mathbb{Z}_{p}$. We fix a topological generator $\gamma$ of $\Gamma$. We recall from $[4, \S 2-\S 3]$ that there are canonical left and right denominator sets $S(G, H)$ and $S(G, H)^{*}$ of $\Lambda(G)$ where

$S(G, H):=\{\lambda \in \Lambda(G): \Lambda(G) /(\Lambda(G) \cdot \lambda)$ is a finitely generated $\Lambda(H)$-module $\}$

and

$$
S(G, H)^{*}:=\bigcup_{i \geq 0} p^{i} S(G, H)
$$

When $G$ and $H$ are clear from context we abbreviate $S(G, H)$ to $S$. We also write $\mathfrak{M}_{S}(G)$ and $\mathfrak{M}_{S^{*}}(G)$ for the categories of finitely generated $\Lambda(G)$-modules $M$ that satisfy $\Lambda(G)_{S} \otimes_{\Lambda(G)} M=0$ and $\Lambda(G)_{S^{*}} \otimes_{\Lambda(G)} M=0$ respectively. We further recall from [4, Prop. 2.3] that a finitely generated $\Lambda(G)$-module $M$ belongs to $\mathfrak{M}_{S}(G)$, resp. to $\mathfrak{M}_{S^{*}}(G)$, if and only if $M$, resp. $M_{\mathrm{tf}}$, is a finitely generated $\Lambda(H)$-module (by restriction). Thus $\mathfrak{M}_{S^{*}}(G)$ coincides with the category $\mathfrak{M}_{H}(G)$ used in [4]. 


\section{$\S 2.3$. Canonical automorphisms}

If $J$ is any profinite group, then we write $M \hat{\otimes}_{\Lambda(J)} N$ for the completed tensor product of compact $\Lambda(J)$-modules $M$ and $N$ (cf. [7, p. 230]). In particular, if $M$ is any compact $\Lambda(G)$-module, then

$$
\mathrm{I}_{H}^{G}(M):=\Lambda(G) \hat{\otimes}_{\Lambda(H)} \operatorname{Res}_{H}^{G}(M)
$$

has a natural structure as a (compact) $\Lambda(G)$-module via left multiplication. The functor $M \mapsto \mathrm{I}_{H}^{G}(M)$ is exact on the category of compact $\Lambda(G)$-modules and if $M$ belongs to $\mathfrak{M}_{S}(G)$, then $\mathrm{I}_{H}^{G}(M)$ identifies with the usual tensor product $\Lambda(G) \otimes_{\Lambda(H)} M$ (cf. [7, p. 241, Ex. 1]).

We define an endomorphism $\Delta_{G, \gamma, M}$ of $\mathrm{I}_{H}^{G}(M)$ by setting

$$
\Delta_{G, \gamma, M}\left(x \otimes_{\Lambda(H)} y\right):=x \tilde{\gamma}^{-1} \otimes_{\Lambda(H)} \tilde{\gamma}(y)
$$

for each $x \in \Lambda(G)$ and $y \in M$, where $\tilde{\gamma}$ is any lift of $\gamma$ through the projection $G \rightarrow \Gamma$. We also set

$$
\delta_{G, \gamma, M}:=\operatorname{id}_{I_{H}^{G}(M)}-\Delta_{G, \gamma, M} .
$$

Then $\Delta_{G, \gamma, M}$, and hence also $\delta_{G, \gamma, M}$, is a well-defined endomorphism of the $\Lambda(G)$-module $\mathrm{I}_{H}^{G}(M)$ that is independent of the precise choice of $\tilde{\gamma}$. When $G$ and $M$ are both clear from context we usually abbreviate $\Delta_{G, \gamma, M}$ and $\delta_{G, \gamma, M}$ to $\Delta_{\gamma}$ and $\delta_{\gamma}$ respectively.

For any compact $\Lambda(G)$-module $M$ we let $M_{S}$, resp. $M_{S^{*}}$, denote the

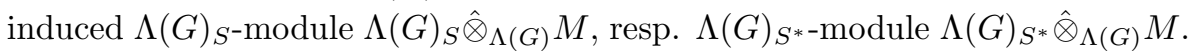

Lemma 2.1. If $\Sigma \in\left\{S, S^{*}\right\}$ and $M \in \mathfrak{M}_{\Sigma}(G)$, then $\mathrm{I}_{H}^{G}(M)_{\Sigma}$ is a finitely generated $\Lambda(G)_{\Sigma}$-module and $\delta_{G, \gamma, M}$ induces an automorphism of $\mathrm{I}_{H}^{G}(M)_{\Sigma}$.

Proof. We assume first that $\Sigma=S$ and fix $M$ in $\mathfrak{M}_{S}(G)$. Then $\mathrm{I}_{H}^{G}(M)$ is a finitely generated $\Lambda(G)$-module and hence $\mathrm{I}_{H}^{G}(M)_{S}$ is a finitely generated $\Lambda(G)_{S}$-module. There is also a short exact sequence of (finitely generated) $\Lambda(G)$-modules

$$
0 \rightarrow \mathrm{I}_{H}^{G}(M) \stackrel{\delta_{\gamma}}{\longrightarrow} \mathrm{I}_{H}^{G}(M) \rightarrow M \rightarrow 0
$$

in which the third arrow is induced by $x \otimes_{\Lambda(H)} m \mapsto x(m)$ for each $x \in \Lambda(G)$ and $m \in M$. Indeed, the exactness of this sequence has been proved by Schneider and Venjakob [8, Prop. 2.2, Rem. 2.3]. Thus, by applying the (exact) 
scalar extension functor $\Lambda(G)_{S} \hat{\otimes}_{\Lambda(G)}$ - to (1) and noting that $M_{S}=0$ (by assumption), we may deduce that $\delta_{\gamma}$ induces an automorphism of $\mathrm{I}_{H}^{G}(M)_{S}$.

From here the analogous results for modules $M$ in $\mathfrak{M}_{S^{*}}(G)$ follow easily from the fact that $M_{\mathrm{tf}}$ belongs to $\mathfrak{M}_{S}(G)$ and $\mathrm{I}_{H}^{G}(M)_{S^{*}}=\mathbb{Q}_{p} \otimes_{\mathbb{Z}_{p}} \mathrm{I}_{H}^{G}\left(M_{\mathrm{tf}}\right)_{S}$.

\section{§2.4. Algebraic $p$-adic $L$-functions}

The ring $\Lambda(G)_{S^{*}}$ is both noetherian and regular [6, Prop. 4.3.4]. Hence there is a natural group isomorphism between the algebraic $K$-group $K_{1}\left(\Lambda(G)_{S^{*}}\right)$ and the group $G_{1}\left(\Lambda(G)_{S^{*}}\right)$ that is generated (multiplicatively) by symbols of the form $\langle\alpha \mid N\rangle$ where $\alpha$ is an automorphism of a finitely generated

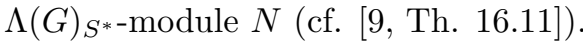

Definition 2.2. Let $A^{\bullet}$ be a complex in $D^{\mathrm{fg}}(\Lambda(G))$ such that $\Lambda(G)_{S} \hat{\otimes}_{\Lambda(G)} A^{\bullet}$ is acyclic. Then (following Lemma 2.1) we define the Algebraic $p$-adic L-function of $A^{\bullet}$ (relative to $G$ and $\gamma$ ) to be the element of $K_{1}\left(\Lambda(G)_{S^{*}}\right)$ obtained by setting

$$
\mathcal{L}_{G, \gamma}^{\mathrm{alg}}\left(A^{\bullet}\right):=\prod_{i \in \mathbb{Z}}\left\langle\delta_{G, \gamma, H^{i}\left(A^{\bullet}\right)} \mid \mathrm{I}_{H}^{G}\left(H^{i}\left(A^{\bullet}\right)\right)_{S^{*}}\right\rangle^{(-1)^{i}} .
$$

The following result explicates this definition in a classical setting.

Lemma 2.3. If $G=\Gamma$, then $\Lambda(G)_{S^{*}}$ is the total quotient ring $Q(\Gamma)$ of $\Lambda(\Gamma)$ and there is a natural isomorphism of groups $\iota: K_{1}\left(\Lambda(G)_{S^{*}}\right) \cong Q(\Gamma)^{\times}$. If $M$ is any $\Lambda(\Gamma)$-module that is finitely generated over $\mathbb{Z}_{p}$, then

$$
\iota\left(\mathcal{L}_{G, \gamma}^{\mathrm{alg}}(M[0])\right)=(1+T)^{-\lambda(M)} \operatorname{char}_{T}(M)
$$

where $\lambda(M)$ is the Iwasawa $\lambda$-invariant of $M$ and $\operatorname{char}_{T}(M)$ the characteristic polynomial of $M$ with respect to the variable $T=\gamma-1$.

Proof. The first sentence is well-known (with the isomorphism $\iota$ induced by taking determinants over $Q(\Gamma)$ ). Further, by the structure theory of finitely generated $\Lambda(\Gamma)$-modules, it suffices to prove the second sentence in the case that $M=\Lambda(\Gamma) /(f)$ where $f$ is a distinguished polynomial. Now $\mathcal{L}_{G, \gamma}^{\text {alg }}(M[0])=$ $\left\langle\delta_{\gamma} \mid Q(\Gamma) \otimes_{\mathbb{Z}_{p}} M\right\rangle$ and so, if we consider $N:=\mathbb{Z}_{p}[[T]] \otimes_{\mathbb{Z}_{p}}\left(\mathbb{Z}_{p}[[T]] /(f(T))\right)$ as a (free) $\mathbb{Z}_{p}[[T]]$-module via left multiplication, then $\iota\left(\mathcal{L}_{G, \gamma}^{\text {alg }}(M[0])\right)=\operatorname{det}_{\mathbb{Z}_{p}[[T]]}(\alpha)$ where $\alpha$ is the endomorphism of $N$ given by multiplication by

$$
\mathrm{id}-(1+T)^{-1} \otimes(1+T)=\left((1+T)^{-1} \otimes \mathrm{id}\right)(T \otimes \mathrm{id}-\mathrm{id} \otimes T) .
$$


But, by using the $\mathbb{Z}_{p}[[T]]$-basis $\left\{1 \otimes\left(T^{i} \bmod (f(T))\right): 0 \leq i<\operatorname{deg}(f)\right\}$ of $N$, one computes that $\operatorname{det}_{\mathbb{Z}_{p}[[T]]}\left((1+T)^{-1} \otimes \mathrm{id} \mid N\right)=(1+T)^{-\operatorname{rank}_{\mathbb{Z}_{p}[[T]]}(N)}=(1+$ $T)^{-\operatorname{deg}(f)}=(1+T)^{-\lambda(M)}$ and $\operatorname{det}_{\mathbb{Z}_{p}[[T]]}(T \otimes \mathrm{id}-\mathrm{id} \otimes T \mid N)=f(T)=\operatorname{char}_{T}(M)$. The displayed equality is therefore clear.

Remark 2.4. In joint work with Venjakob [3] we reinterpret Definition 2.2 in terms of the localized $K_{1}$-groups introduced by Fukaya and Kato in [6]. If $G$ has no element of order $p$, then in [3] we also extend Definition 2.2 (and the results proved in $\S 3$ and $\S 4$ below) to the case of complexes $A^{\bullet}$ in $D^{\mathrm{fg}}(\Lambda(G))$ for which only $\Lambda(G)_{S^{*}} \hat{\otimes}_{\Lambda(G)} A^{\bullet}$ is assumed to be acyclic.

\section{$\S 3 . \quad$ Basic Properties}

If $\Sigma$ denotes either $S$ or $S^{*}$, then we write $D_{\Sigma}^{\mathrm{p}}(\Lambda(G))$ for the full triangulated subcategory of $D^{\mathrm{p}}(\Lambda(G))$ comprising complexes $A^{\bullet}$ in $D^{\mathrm{p}}(\Lambda(G))$ for which $\Lambda(G)_{\Sigma} \hat{\otimes}_{\Lambda(G)} A^{\bullet}$ is acyclic. For each $M$ in $\mathfrak{M}_{S^{*}}(G)$ we also set

$$
\mathcal{E}_{G, \gamma}(M):=\left\langle\delta_{G, \gamma, M} \mid \mathrm{I}_{H}^{G}(M)_{S^{*}}\right\rangle \in K_{1}\left(\Lambda(G)_{S^{*}}\right) .
$$

Proposition 3.1. (Additivity) If $A^{\bullet} \rightarrow B^{\bullet} \rightarrow C^{\bullet} \rightarrow A^{\bullet}[1]$ is an exact triangle in $D_{S}^{\mathrm{p}}(\Lambda(G))$, then $\mathcal{L}_{G, \gamma}^{\mathrm{alg}}\left(B^{\bullet}\right)=\mathcal{L}_{G, \gamma}^{\mathrm{alg}}\left(A^{\bullet}\right) \mathcal{L}_{G, \gamma}^{\mathrm{alg}}\left(C^{\bullet}\right)$.

Proof. Applying the exact functor $\mathrm{I}_{H}^{G}(-)_{S^{*}}$ to the long exact cohomology sequence of the given triangle gives a commutative diagram of finitely generated $\Lambda(G)_{S^{*} \text {-modules }}$

$$
\begin{aligned}
& \rightarrow \mathrm{I}_{H}^{G}\left(H^{i}\left(A^{\bullet}\right)\right)_{S^{*}} \rightarrow \mathrm{I}_{H}^{G}\left(H^{i}\left(B^{\bullet}\right)\right)_{S^{*}} \rightarrow \mathrm{I}_{H}^{G}\left(H^{i}\left(C^{\bullet}\right)\right)_{S^{*}} \rightarrow \mathrm{I}_{H}^{G}\left(H^{i+1}\left(A^{\bullet}\right)\right)_{S^{*}} \rightarrow \\
& \delta_{G, \gamma, H^{i}\left(A^{\bullet}\right) \downarrow} \downarrow \delta_{G, \gamma, H^{i}\left(B^{\bullet}\right)} \downarrow \quad \delta_{G, \gamma, H^{i}\left(C^{\bullet}\right)} \downarrow \quad \delta_{G, \gamma, H^{i+1}\left(A^{\bullet}\right) \downarrow} \downarrow \\
& \rightarrow \mathrm{I}_{H}^{G}\left(H^{i}\left(A^{\bullet}\right)\right)_{S^{*}} \rightarrow \mathrm{I}_{H}^{G}\left(H^{i}\left(B^{\bullet}\right)\right)_{S^{*}} \rightarrow \mathrm{I}_{H}^{G}\left(H^{i}\left(C^{\bullet}\right)\right)_{S^{*}} \rightarrow \mathrm{I}_{H}^{G}\left(H^{i+1}\left(A^{\bullet}\right)\right)_{S^{*}} \rightarrow
\end{aligned}
$$

in which both rows are exact. Taken in conjunction with the defining relations of $K_{1}\left(\Lambda(G)_{S^{*}}\right)$ this diagram implies the required equality

$$
\begin{aligned}
\mathcal{L}_{G, \gamma}^{\mathrm{alg}}\left(B^{\bullet}\right) & =\prod_{i \in \mathbb{Z}} \mathcal{E}_{G, \gamma}\left(H^{i}\left(B^{\bullet}\right)\right)^{(-1)^{i}} \\
= & \prod_{i \in \mathbb{Z}} \mathcal{E}_{G, \gamma}\left(H^{i}\left(A^{\bullet}\right)\right)^{(-1)^{i}} \prod_{i \in \mathbb{Z}} \mathcal{E}_{G, \gamma}\left(H^{i}\left(C^{\bullet}\right)\right)^{(-1)^{i}}=\mathcal{L}_{G, \gamma}^{\mathrm{alg}}\left(A^{\bullet}\right) \mathcal{L}_{G, \gamma}^{\mathrm{alg}}\left(C^{\bullet}\right) .
\end{aligned}
$$


Now let $U$ be a closed subgroup of $H$ that is normal in $G$ and set $\bar{G}:=$ $G / U, \bar{H}:=H / U$ and $\bar{S}:=S(\bar{G}, \bar{H})$. Then the natural projection $\Lambda(G) \rightarrow$ $\Lambda(\bar{G})$ extends to a ring homomorphism $\Lambda(G)_{S^{*}} \rightarrow \Lambda(\bar{G})_{S^{*}}$ and hence induces a homomorphism of groups $\pi_{\bar{G}}: K_{1}\left(\Lambda(G)_{S^{*}}\right) \rightarrow K_{1}\left(\Lambda(\bar{G})_{S^{*}}\right)$.

Proposition 3.2. $\quad$ (Change of group) If $A^{\bullet}$ belongs to $D_{S}^{\mathrm{p}}(\Lambda(G)$ ), then $\Lambda(\bar{G}) \hat{\otimes}_{\Lambda(G)}^{\mathbb{L}} A^{\bullet}$ belongs to $D_{\bar{S}}^{\mathrm{p}}(\Lambda(\bar{G}))$ and $\pi_{\bar{G}}\left(\mathcal{L}_{G, \gamma}^{\text {alg }}\left(A^{\bullet}\right)\right)=\mathcal{L}_{\bar{G}, \gamma}^{\text {alg }}\left(\Lambda(\bar{G}) \hat{\otimes}_{\Lambda(G)}^{\mathbb{L}} A^{\bullet}\right)$.

Proof. The first assertion follows directly from the fact that there is a natural isomorphism in $D^{\mathrm{p}}\left(\Lambda(\bar{G})_{\bar{S}}\right)$ of the form

$$
\Lambda(\bar{G})_{\bar{S}} \hat{\otimes}_{\Lambda(\bar{G})}\left(\Lambda(\bar{G}) \hat{\otimes}_{\Lambda(G)}^{\mathbb{L}} A^{\bullet}\right) \cong \Lambda(\bar{G})_{\bar{S}} \hat{\otimes}_{\Lambda(G)_{S}}^{\mathbb{L}}\left(\Lambda(G)_{S} \hat{\otimes}_{\Lambda(G)} A^{\bullet}\right) .
$$

To prove the second assertion we first recall that each term $\mathcal{E}_{G, \gamma}\left(H^{i}\left(A^{\bullet}\right)\right)$ can be computed explicitly as follows. One can fix a complex $P_{i}^{\bullet}$ in $C^{\mathrm{p}}\left(\Lambda(G)_{S^{*}}\right)$, an isomorphism $\psi_{i}: P_{i}^{\bullet} \rightarrow \mathrm{I}_{H}^{G}\left(H^{i}\left(A^{\bullet}\right)\right)_{S^{*}}[0]$ in $D^{\mathrm{p}}\left(\Lambda(G)_{S^{*}}\right)$ and a morphism $\alpha_{i}: P_{i}^{\bullet} \rightarrow P_{i}^{\bullet}$ in $C^{\mathrm{p}}\left(\Lambda(G)_{S^{*}}\right)$ which is bijective in each degree and such that the following diagram commutes in $D^{\mathrm{p}}\left(\Lambda(G)_{S^{*}}\right)$

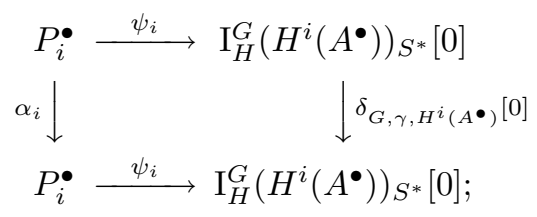

one then has $\mathcal{E}_{G, \gamma}\left(H^{i}\left(A^{\bullet}\right)\right)=\prod_{j \in \mathbb{Z}}\left\langle\alpha_{i}^{j} \mid P_{i}^{j}\right\rangle^{(-1)^{j}}$. Hence

$$
\begin{aligned}
\pi_{\bar{G}}\left(\mathcal{L}_{G, \gamma}^{\mathrm{alg}}\left(A^{\bullet}\right)\right) & =\prod_{i \in \mathbb{Z}} \pi_{\bar{G}}\left(\mathcal{E}_{G, \gamma}\left(H^{i}\left(A^{\bullet}\right)\right)^{(-1)^{i}}\right. \\
& =\prod_{i \in \mathbb{Z}} \prod_{j \in \mathbb{Z}}\left\langle\mathrm{id} \otimes \alpha_{i}^{j} \mid \Lambda(\bar{G})_{\bar{S}^{*}} \otimes_{\Lambda(G)_{S^{*}}} P_{i}^{j}\right\rangle^{(-1)^{i+j}} \\
& =\prod_{i \in \mathbb{Z}} \prod_{j \in \mathbb{Z}}\left\langle H^{j}\left(\mathrm{id} \otimes \alpha_{i}\right) \mid H^{j}\left(\Lambda(\bar{G})_{\bar{S}^{*}} \otimes_{\Lambda(G)_{S^{*}}} P_{i}^{\bullet}\right)\right\rangle^{(-1)^{i+j}}
\end{aligned}
$$

where the last equality follows from the regularity of $\Lambda(\bar{G})_{\bar{S}^{*}}$ and the defining relations of $K_{1}\left(\Lambda(\bar{G})_{\bar{S}^{*}}\right)$. Now there are natural isomorphisms of $\Lambda(\bar{G})_{\bar{S}^{*-}}$ modules

$$
H^{j}\left(\Lambda(\bar{G})_{\bar{S}^{*}} \otimes_{\Lambda(G)_{S^{*}}} P_{i}^{\bullet}\right) \cong \mathrm{I} \frac{\bar{G}}{H}\left(\operatorname{Tor}_{j}^{\Lambda(G)}\left(\Lambda(\bar{G}), H^{i}\left(A^{\bullet}\right)\right)\right)_{\bar{S}^{*}}
$$

under which $H^{j}\left(\mathrm{id} \otimes \alpha_{i}\right)$ corresponds to the endomorphism $\delta_{\bar{G}, \gamma, \operatorname{Tor}_{j}^{\Lambda}}^{\Lambda(G)}\left(\Lambda(\bar{G}), H^{i}(A \bullet)\right)^{\bullet}$ 
The product expression (2) is therefore equal to

$$
\begin{aligned}
& \prod_{i \in \mathbb{Z}} \prod_{j \in \mathbb{Z}}\left\langle\delta_{\bar{G}, \gamma, \operatorname{Tor}_{j}^{\Lambda(G)}\left(\Lambda(\bar{G}), H^{i}\left(A^{\bullet}\right)\right)} \mid \mathrm{I}_{\bar{H}}^{\bar{G}}\left(\operatorname{Tor}_{j}^{\Lambda(G)}\left(\Lambda(\bar{G}), H^{i}\left(A^{\bullet}\right)\right)\right)_{\bar{S}^{*}}\right\rangle^{(-1)^{i+j}} \\
= & \prod_{i \in \mathbb{Z}}\left\langle\delta_{\bar{G}, \gamma, H^{i}\left(\Lambda(\bar{G}) \hat{\otimes}_{\Lambda(G)}^{\mathbb{L}} A^{\bullet}\right)} \mid \mathrm{I}_{\bar{H}}^{\bar{G}}\left(H^{i}\left(\Lambda(\bar{G}) \hat{\otimes}_{\Lambda(G)}^{\mathbb{L}} A^{\bullet}\right)\right)_{\bar{S}^{*}}\right\rangle^{(-1)^{i}} \\
= & \prod_{i \in \mathbb{Z}} \mathcal{E}_{\bar{G}, \gamma}\left(H^{i}\left(\Lambda(\bar{G}) \hat{\otimes}_{\Lambda(G)}^{\mathbb{L}} A^{\bullet}\right)\right)^{(-1)^{i}} \\
= & \mathcal{L}_{\bar{G}, \gamma}^{\mathrm{alg}}\left(\Lambda(\bar{G}) \hat{\otimes}_{\Lambda(G)}^{\mathbb{L}} A^{\bullet}\right)
\end{aligned}
$$

where the first displayed equality is a consequence of the spectral sequence $E_{2}^{r, s}=\operatorname{Tor}_{r}^{\Lambda(G)}\left(\Lambda(\bar{G}), H^{s}\left(A^{\bullet}\right)\right) \Longrightarrow H^{s-r}\left(\Lambda(\bar{G}) \hat{\otimes}_{\Lambda(G)}^{\mathbb{L}} A^{\bullet}\right)$.

Remark 3.3. (Akashi series) If $G$ has no element of order $p$, then for each module $M$ in $\mathfrak{M}_{S}(G)$ the complex $M[0]$ belongs to $D_{S}^{\mathrm{p}}(\Lambda(G))$. Proposition 3.2 (with $\bar{G}=\Gamma$ ) thus combines with Lemma 2.3 to imply that the composite homomorphism $K_{1}(Q(\Gamma)) \cong Q(\Gamma)^{\times} \rightarrow Q(\Gamma)^{\times} / \Lambda(\Gamma)^{\times}$sends the element $\pi_{\Gamma}\left(\mathcal{L}_{G, \gamma}^{\mathrm{alg}}(M[0])\right)=\mathcal{L}_{\Gamma, \gamma}^{\mathrm{alg}}\left(\Lambda(\Gamma) \hat{\otimes}_{\Lambda(G)}^{\mathbb{L}} M[0]\right)$ to the 'Akashi series' $f_{M}$ of $M$ that is introduced by Coates, Schneider and Sujatha in [5, §4] (and is denoted by $\operatorname{Ak}(M)$ in $[4, \S 3])$.

\section{§4. Characteristic Elements}

We write $G_{0}\left(\mathfrak{M}_{S^{*}}(G)\right)$ for the Grothendieck group of the category $\mathfrak{M}_{S^{*}}(G)$ and for each module $M$ in $\mathfrak{M}_{S^{*}}(G)$ we write $[M]$ for the associated element of $G_{0}\left(\mathfrak{M}_{S^{*}}(G)\right)$. We also write $K_{0}\left(\Lambda(G), \Lambda(G)_{S^{*}}\right)$ for the relative algebraic $K_{0}$-group of the natural homomorphism $\Lambda(G) \rightarrow \Lambda(G)_{S^{*}}$ and recall that this group is generated by triples of the form $(P, \kappa, Q)$ where $P$ and $Q$ are finitely generated projective $\Lambda(G)$-modules and $\kappa$ is an isomorphism of $\Lambda(G)_{S^{*} \text {-modules }}$ $P_{S^{*}} \cong Q_{S^{*}}$ (for further details see $[9$, p. 215]).

If $G$ has no element of order $p$, then $\Lambda(G)$ is a noetherian regular ring and the groups $K_{0}\left(\Lambda(G), \Lambda(G)_{S^{*}}\right)$ and $G_{0}\left(\mathfrak{M}_{S^{*}}(G)\right)$ are naturally isomorphic. We normalise this isomorphism in the following way: if $g=s^{-1} h$ with $s \in S^{*}$ and $h \in \Lambda(G) \cap \Lambda(G)_{S^{*}}^{\times}$, then the element $\left(\Lambda(G), \mathrm{r}_{g}, \Lambda(G)\right)$ of $K_{0}\left(\Lambda(G), \Lambda(G)_{S^{*}}\right)$ corresponds to the element $\left[\operatorname{cok}\left(\mathrm{r}_{h}\right)\right]-\left[\operatorname{cok}\left(\mathrm{r}_{s}\right)\right]$ of $G_{0}\left(\mathfrak{M}_{S^{*}}(G)\right)$ where $\mathrm{r}_{g}, \mathrm{r}_{h}$ and $\mathrm{r}_{s}$ denote the automorphisms of $\Lambda(G)_{S^{*}}$ that are induced by right multiplication by $g, h$ and $s$ respectively.

We next note that, irrespective of whether $G$ has an element of order $p$, each complex $A^{\bullet}$ in $D_{S^{*}}^{\mathrm{p}}(\Lambda(G))$ gives rise to a canonical 'euler character- 
istic' element $\chi\left(A^{\bullet}\right)$ in $K_{0}\left(\Lambda(G), \Lambda(G)_{S^{*}}\right)$. We define this element by identifying $K_{0}\left(\Lambda(G), \Lambda(G)_{S^{*}}\right)$ with $\pi_{0}$ of a natural Picard category that is constructed from the categories of virtual objects $\mathcal{V}(\Lambda(G))$ and $\mathcal{V}\left(\Lambda(G)_{S^{*}}\right)$ associated to the categories of finitely generated projective $\Lambda(G)$-modules and

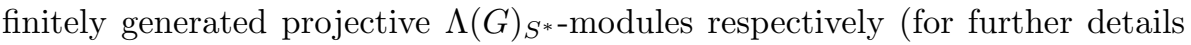
see, for example, [1, Lem. 5.1]). Then, with respect to this identification, we let $\chi\left(A^{\bullet}\right)$ denote the inverse of the element of $K_{0}\left(\Lambda(G), \Lambda(G)_{S^{*}}\right)$ that corresponds to the pair $\left(\left[P^{\bullet}\right], \iota_{P} \bullet\right)$ where $\left[P^{\bullet}\right]$ is the object of $\mathcal{V}(\Lambda(G))$ associated to any $P^{\bullet}$ in $C^{\mathrm{p}}(\Lambda(G))$ that is isomorphic in $D^{\mathrm{p}}(\Lambda(G))$ to $A^{\bullet}$ and $\iota_{P} \bullet$ is the morphism in $\mathcal{V}\left(\Lambda(G)_{S^{*}}\right)$ associated to the isomorphism $\Lambda(G)_{S^{*}} \otimes_{\Lambda(G)} P^{\bullet} \cong$ $\Lambda(G)_{S^{*}} \hat{\otimes}_{\Lambda(G)} P^{\bullet} \cong \Lambda(G)_{S^{*}} \hat{\otimes}_{\Lambda(G)} A^{\bullet} \cong 0$ in $D^{\mathrm{p}}\left(\Lambda(G)_{S^{*}}\right)$. This element $\chi\left(A^{\bullet}\right)$

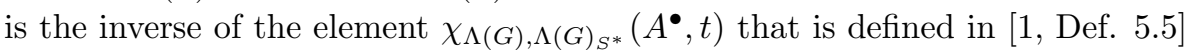
with $t$ equal to the isomorphism $\bigoplus_{i \in \mathbb{Z}} H^{2 i}\left(A^{\bullet}\right)_{S^{*}} \cong 0 \cong \bigoplus_{i \in \mathbb{Z}} H^{2 i+1}\left(A^{\bullet}\right)_{S^{*}}$. (We prefer to define $\chi\left(A^{\bullet}\right)$ in terms of the inverse in order to ensure that if $G$ has no element of order $p$ and $M$ belongs to $\mathfrak{M}_{S^{*}}(G)$, then the isomorphism $K_{0}\left(\Lambda(G), \Lambda(G)_{S^{*}}\right) \cong G_{0}\left(\mathfrak{M}_{S^{*}}(G)\right)$ described above sends $\chi(M[0])$ to $[M]$.)

In the next result we use the natural connecting homomorphisms in $K$ theory

$$
\partial_{G}^{\prime}: K_{1}\left(\Lambda(G)_{S^{*}}\right) \cong G_{1}\left(\Lambda(G)_{S^{*}}\right) \rightarrow G_{0}\left(\mathfrak{M}_{S^{*}}(G)\right)
$$

and

$$
\partial_{G}: K_{1}\left(\Lambda(G)_{S^{*}}\right) \rightarrow K_{0}\left(\Lambda(G), \Lambda(G)_{S^{*}}\right) .
$$

Theorem 4.1. Let $A^{\bullet}$ be a complex in $D_{S}^{\mathrm{p}}(\Lambda(G))$.

(i) $\partial_{G}^{\prime}\left(\mathcal{L}_{G, \gamma}^{\mathrm{alg}}\left(A^{\bullet}\right)\right)=\sum_{i \in \mathbb{Z}}(-1)^{i}\left[H^{i}\left(A^{\bullet}\right)\right]$.

(ii) If $G$ has rank one (as a p-adic Lie group), then $\partial_{G}\left(\mathcal{L}_{G, \gamma}^{\text {alg }}\left(A^{\bullet}\right)\right)=\chi\left(A^{\bullet}\right)$.

Remark 4.2. (Characteristic elements) In the setting of Lemma 2.3 claim (i) of this result recovers the fact that $\operatorname{char}_{T}(M)$ generates the characteristic ideal of $M$. More generally, claim (i) implies that if $G$ has no element of order $p$, then $\mathcal{L}_{G, \gamma}^{\text {alg }}\left(A^{\bullet}\right)$ is a 'characteristic element of $A^{\bullet}$ ' in the sense of [4, (33)]. (If $G$ has rank one, then) the equality of claim (ii) is in general much finer than that of claim (i) and plays a key role in the descent theory formulated in [3].

\section{§4.1. The proof of Theorem 4.1(i)}

We write $\partial_{G}^{\prime \prime}: G_{1}\left(\Lambda(G)_{S^{*}}\right) \rightarrow G_{0}\left(\mathfrak{M}_{S^{*}}(G)\right)$ for the natural connecting homomorphism (that occurs in the above definition of $\partial_{G}^{\prime}$ ) and recall that if $\nu$ 
is an automorphism of a finitely generated $\Lambda(G)_{S^{*}-\text { module }} N$, then

$$
\partial_{G}^{\prime \prime}(\langle\nu, N\rangle)=[\mathcal{N} /(\mathcal{N} \cap \nu(\mathcal{N}))]-[\nu(\mathcal{N}) /(\mathcal{N} \cap \nu(\mathcal{N}))]
$$

where $\mathcal{N}$ is any finitely generated $\Lambda(G)$-submodule of $N$ with $\mathcal{N}_{S^{*}}=N$.

For each integer $i$ we set $M^{i}:=H^{i}\left(A^{\bullet}\right)$. Then Lemma 4.3(ii) below (with $M=M^{i}$ ) implies that the (finitely generated) $\Lambda(G)$-module $\mathrm{I}_{H}^{G}\left(M_{\mathrm{tf}}^{i}\right)$ is isomorphic to its image in $\mathrm{I}_{H}^{G}\left(M^{i}\right)_{S^{*}}$. Hence, if we set $\delta_{\gamma}^{i}:=\delta_{G, \gamma, M^{i}}$, then in $G_{0}\left(\mathfrak{M}_{S^{*}}(G)\right)$ one has

$$
\begin{aligned}
\partial_{G}^{\prime}\left(\mathcal{L}_{G, \gamma}^{\mathrm{alg}}\left(A^{\bullet}\right)\right) & =\sum_{i \in \mathbb{Z}}(-1)^{i} \partial_{G}^{\prime \prime}\left(\left\langle\delta_{\gamma}^{i} \mid \mathrm{I}_{H}^{G}\left(M^{i}\right)_{S^{*}}\right\rangle\right)=\sum_{i \in \mathbb{Z}}(-1)^{i}\left[\mathrm{I}_{H}^{G}\left(M_{\mathrm{tf}}^{i}\right) / \delta_{\gamma}^{i}\left(\mathrm{I}_{H}^{G}\left(M_{\mathrm{tf}}^{i}\right)\right)\right] \\
& =\sum_{i \in \mathbb{Z}}(-1)^{i}\left[M_{\mathrm{tf}}^{i}\right]=\sum_{i \in \mathbb{Z}}(-1)^{i}\left(\left[M^{i}\right]-\left[M_{\mathrm{tor}}^{i}\right]\right) .
\end{aligned}
$$

Here the second equality follows from (3) (with $\mathcal{N}=\mathrm{I}_{H}^{G}\left(M_{\mathrm{tf}}^{i}\right)$ and $\nu=\delta_{\gamma}^{i}$ ) and the fact that $\delta_{\gamma}^{i}\left(\mathrm{I}_{H}^{G}\left(M_{\mathrm{tf}}^{i}\right)\right) \subseteq \mathrm{I}_{H}^{G}\left(M_{\mathrm{tf}}^{i}\right)$ and the third from the isomorphism $\mathrm{I}_{H}^{G}\left(M_{\mathrm{tf}}^{i}\right) / \delta_{\gamma}^{i}\left(\mathrm{I}_{H}^{G}\left(M_{\mathrm{tf}}^{i}\right)\right) \cong M_{\mathrm{tf}}^{i}$ that is induced by (1) with $M=M_{\mathrm{tf}}^{i}$. Given the last displayed formula, the proof of Theorem 4.1(i) is therefore completed by applying Lemma 4.3(i) below with $M=M^{i}$ (for each $i$ ).

\section{Lemma 4.3.}

(i) If $M$ belongs to $\mathfrak{M}_{S}(G)$, then $\left[M_{\text {tor }}\right]=0$ in $G_{0}\left(\mathfrak{M}_{S^{*}}(G)\right)$.

(ii) If $M$ belongs to $\mathfrak{M}_{S^{*}}(G)$, then the natural map $\mathrm{I}_{H}^{G}\left(M_{\mathrm{tf}}\right) \rightarrow \mathrm{I}_{H}^{G}(M)_{S^{*}}$ is injective.

Proof. Claim (i) follows from the fact that if $M$ is in $\mathfrak{M}_{S}(G)$, then (1) with $M=M_{\text {tor }}$ is a short exact sequence of objects of $\mathfrak{M}_{S^{*}}(G)$ and hence implies that $\left[M_{\text {tor }}\right]=\left[\mathrm{I}_{H}^{G}\left(M_{\text {tor }}\right)\right]-\left[\mathrm{I}_{H}^{G}\left(M_{\text {tor }}\right)\right]=0$ in $G_{0}\left(\mathfrak{M}_{S^{*}}(G)\right)$.

To prove claim (ii) we note that $\mathrm{I}_{H}^{G}\left(M_{\mathrm{tf}}\right)$ is a finitely generated $\Lambda(G)$ module that is $\mathbb{Z}_{p}$-torsion-free (and hence that a $\Lambda(G)$-submodule of $\mathrm{I}_{H}^{G}\left(M_{\mathrm{tf}}\right)$ belongs to $\mathfrak{M}_{S^{*}}(G)$ if and only if it belongs to $\mathfrak{M}_{S}(G)$ ). It therefore suffices to prove that if $M$ is any $\mathbb{Z}_{p}$-torsion-free module in $\mathfrak{M}_{S}(G)$ and $N$ a $\Lambda(G)$ submodule of $\mathrm{I}_{H}^{G}(M)$ that is finitely generated over $\Lambda(H)$, then $N=0$. To do this we fix a pro- $p$ open subgroup $J$ of $H$ that is normal in $G$. We write $I(J)$ for the kernel of the projection map $\Lambda(G) \rightarrow \Lambda(G / J)$ (so $\left.M_{J} \cong M / I(J) M\right)$ and let $\bar{N}$ denote the image of $N$ under the canonical projection $\mathrm{I}_{H}^{G}(M) \rightarrow$ $\mathrm{I}_{H}^{G}\left(M_{J}\right) \cong \mathrm{I}_{H / J}^{G / J}\left(M_{J}\right)$.

Now if $\tilde{\Gamma}$ denotes the subgroup of $G / J$ that is generated topologically by a choice of pre-image $\tilde{\gamma}$ of $\gamma$ under the surjection $G / J \rightarrow \Gamma$, then $\mathrm{I}_{H / J}^{G / J}\left(M_{J}\right)$ is 
isomorphic as a $\Lambda(\tilde{\Gamma})$-module to $\Lambda(\tilde{\Gamma}) \otimes_{\mathbb{Z}_{p}} M_{J}$ and $\bar{N}$ identifies with a $\Lambda(\tilde{\Gamma})$ submodule of $\Lambda(\tilde{\Gamma}) \otimes_{\mathbb{Z}_{p}} M_{J}$ that is finitely generated over $\Lambda(H / J)=\mathbb{Z}_{p}[H / J]$ and hence also over $\mathbb{Z}_{p}$. We set $t:=\tilde{\gamma}-1 \in \Lambda(\tilde{\Gamma})$. Now $\bar{N}_{\text {tor }}$ is a finite $\Lambda(\tilde{\Gamma})$ submodule of $\Lambda(\tilde{\Gamma}) \otimes_{\mathbb{Z}_{p}} M_{J \text {,tor }} \subseteq \Lambda(\tilde{\Gamma}) \otimes_{\mathbb{Z}_{p}} M_{J}$ and so if $x \in \bar{N}_{\text {tor }}$, then there exist integers $r>s>0$ such that $t^{r} x=t^{s} x$. But every element of $\Lambda(\tilde{\Gamma}) \otimes_{\mathbb{Z}_{p}} M_{J \text {,tor }}$ can be written uniquely in the form $\sum_{a \geq 0} t^{a} \otimes y_{a}$ with $y_{a} \in M_{J \text {,tor }}$ and so $t^{r} x=t^{s} x$ implies that $x=0$. It follows that $\bar{N}_{\text {tor }}=0$ and hence we can regard $\bar{N}$ as a $\Lambda(\tilde{\Gamma})$-submodule of $\Lambda(\tilde{\Gamma}) \otimes_{\mathbb{Z}_{p}} M_{J, t \mathrm{ff}}$ which is itself finitely generated over $\mathbb{Z}_{p}$. But $M_{J, \mathrm{tf}}$ is a free $\mathbb{Z}_{p}$-module so $\Lambda(\tilde{\Gamma}) \otimes_{\mathbb{Z}_{p}} M_{J, \mathrm{tf}}$ is a free $\Lambda(\tilde{\Gamma})$-module and hence cannot contain any non-zero $\Lambda(\tilde{\Gamma})$-submodule which is finitely generated over $\mathbb{Z}_{p}$. Hence $\bar{N}=0$. From the exact sequence $0 \rightarrow \mathrm{I}_{H}^{G}(I(J) M) \rightarrow \mathrm{I}_{H}^{G}(M) \rightarrow$ $\mathrm{I}_{H}^{G}\left(M_{J}\right) \rightarrow 0$ we therefore deduce that $N \subseteq \mathrm{I}_{H}^{G}(I(J) M)$.

By successively repeating the above argument with $M$ replaced by $I(J) M$, then $I(J)^{2} M$ etc., we deduce that $N$ is contained in $\bigcap_{k \geq 0} \mathrm{I}_{H}^{G}\left(I(J)^{k} M\right)$. Now $I(J)^{k^{\prime}} M \subseteq I(J)^{k} M$ and hence also $\mathrm{I}_{H}^{G}\left(I(J)^{k^{\prime}} M\right) \subseteq \mathrm{I}_{H}^{G}\left(I(J)^{k} M\right)$ for each $k^{\prime} \geq$ $k$ and so the intersections $\bigcap_{k>0} I(J)^{k} M$ and $\bigcap_{k \geq 0} \mathrm{I}_{H}^{G}\left(I(J)^{k} M\right)$ can both be computed as inverse limits. Since completed tensor products commute with inverse limits it follows that $\bigcap_{k \geq 0} \mathrm{I}_{H}^{G}\left(I(J)^{k} M\right)=\mathrm{I}_{H}^{G}\left(\bigcap_{k \geq 0} I(J)^{k} M\right)$. But $I(J)$ is contained in the radical of $\Lambda(G)$ (since $J$ is pro-p) and so $\bigcap_{k \geq 0} I(J)^{k} M=0$ (cf. [7, Prop. (5.2.17)]). Hence $N=0$, as required.

\section{$\S 4.2$. The proof of Theorem 4.1 (ii)}

In this subsection we assume that $G$ has rank one as a $p$-adic Lie group and hence that $\Lambda(G)_{S^{*}}$ is equal to the semisimple artinian ring $Q(G)$. We note first that since $A^{\bullet}$ belongs to $D_{S}^{\mathrm{p}}(\Lambda(G))$ the complex $\mathrm{I}_{H}^{G}\left(A^{\bullet}\right)$ belongs to $D^{\mathrm{p}}(\Lambda(G))$. We may thus choose a complex $P^{\bullet}$ in $C^{\mathrm{p}}(\Lambda(G))$ for which there exists an isomorphism $\psi: P^{\bullet} \stackrel{\sim}{\longrightarrow} \mathrm{I}_{H}^{G}\left(A^{\bullet}\right)$ in $D^{\mathrm{p}}(\Lambda(G))$ and a morphism $\alpha: P^{\bullet} \rightarrow P^{\bullet}$ in $C^{\mathrm{p}}(\Lambda(G))$ such that the following diagram commutes in $D^{\mathrm{p}}(\Lambda(G))$

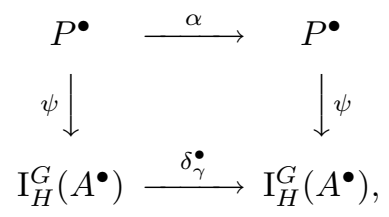

where $\delta_{\gamma}^{\bullet}$ is the morphism with $\delta_{\gamma}^{i}=\delta_{G, \gamma, A^{i}}$ in each degree $i$. Now $H^{i}\left(\mathrm{I}_{H}^{G}\left(A^{\bullet}\right)\right)=$ $\mathrm{I}_{H}^{G}\left(H^{i}\left(A^{\bullet}\right)\right)$ and so (1) with $M=H^{i}\left(A^{\bullet}\right)$ implies that $H^{i}\left(\delta_{\gamma}^{\bullet}\right)$ (and therefore also $\left.H^{i}(\alpha)\right)$ is injective in each degree $i$. Hence, by using Lemma 4.4 below, we 
may change $\alpha$ by a homotopy in order to assume that $\alpha^{i}$ is itself injective in each degree $i$. Thus there exists a short exact sequence of (bounded) complexes of finitely generated $\Lambda(G)$-modules

$$
0 \rightarrow P^{\bullet} \stackrel{\alpha}{\rightarrow} P^{\bullet} \rightarrow \operatorname{cok}(\alpha)^{\bullet} \rightarrow 0
$$

where $\operatorname{cok}(\alpha)^{i}=\operatorname{cok}\left(\alpha^{i}\right)$ in each degree $i$ and the differentials of $\operatorname{cok}(\alpha)^{\bullet}$ are induced by those of $P^{\bullet}$. This sequence implies that for each $i$ the complex $\operatorname{cok}(\alpha)^{i}[-i]$ is naturally quasi-isomorphic to $P^{i} \stackrel{\alpha^{i}}{\longrightarrow} P^{i}$, where the first term occurs in degree $i-1$, and hence both belongs to $D_{S^{*}}^{\mathrm{p}}(\Lambda(G))$ and also satisfies

$$
\chi\left(\operatorname{cok}(\alpha)^{i}[-i]\right)=(-1)^{i}\left(P^{i}, \alpha^{i}, P^{i}\right)=\partial_{G}\left(\left\langle\alpha^{i} \mid P_{S^{*}}^{i}\right\rangle^{(-1)^{i}}\right),
$$

where the first equality is a consequence of our chosen normalisation of $\chi(-)$ and the second a consequence of the definition of $\partial_{G}$.

Next we combine the exact sequence (5) with the commutativity of (4) to deduce that $\operatorname{cok}(\alpha)^{\bullet}$ is isomorphic in $D^{\mathrm{p}}(\Lambda(G))$ to the mapping cone cone $\left(\delta_{\gamma}^{\bullet}\right)$ of $\delta_{\gamma}^{\bullet}$. On the other hand, by using the exact sequences (1) with $M=H^{i}\left(A^{\bullet}\right)$ for each $i$, it is straightforward to show that the morphism cone $\left(\delta_{\gamma}^{\bullet}\right) \rightarrow A^{\bullet}$ which, in each degree $i$, sends $\left(x^{i}, x^{i+1}\right) \in \mathrm{I}_{H}^{G}\left(A^{i}\right) \oplus \mathrm{I}_{H}^{G}\left(A^{i+1}\right)=\operatorname{cone}\left(\delta_{\gamma}^{\bullet}\right)^{i}$ to the image of $x^{i}$ under the natural map $\mathrm{I}_{H}^{G}\left(A^{i}\right) \rightarrow A^{i}$ is a quasi-isomorphism. It follows that $\operatorname{cok}(\alpha)^{\bullet}$ is isomorphic in $D^{\mathrm{p}}(\Lambda(G))$ to $A^{\bullet}$ and hence that $\chi\left(A^{\bullet}\right)=\chi\left(\operatorname{cok}(\alpha)^{\bullet}\right)$ by [1, Prop. 5.6]. Now in each degree $i$ there is an exact sequence of complexes $0 \rightarrow \operatorname{cok}(\alpha)^{i}[-i] \rightarrow \tau_{\leq i}\left(\operatorname{cok}(\alpha)^{\bullet}\right) \rightarrow \tau_{\leq i-1}\left(\operatorname{cok}(\alpha)^{\bullet}\right) \rightarrow 0$ where $\tau_{\leq d}$ denotes naive truncation in degree $d$. By applying [1, Th. 5.7] to each of these exact sequences we obtain an equality $\chi\left(\operatorname{cok}(\alpha)^{\bullet}\right)=\sum_{i \in \mathbb{Z}} \chi\left(\operatorname{cok}(\alpha)^{i}[-i]\right)$ and hence

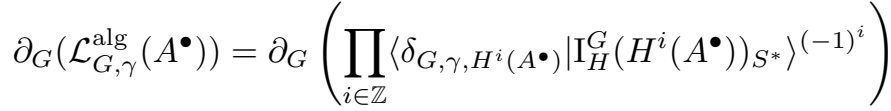

$$
\begin{aligned}
& =\partial_{G}\left(\prod_{i \in \mathbb{Z}}\left\langle H^{i}(\alpha) \mid H^{i}\left(P^{\bullet}\right)_{S^{*}}\right\rangle^{(-1)^{i}}\right) \\
& =\partial_{G}\left(\prod_{i \in \mathbb{Z}}\left\langle\alpha^{i} \mid P_{S^{*}}^{i}\right\rangle^{(-1)^{i}}\right) \\
& =\sum_{i \in \mathbb{Z}} \chi\left(\operatorname{cok}(\alpha)^{i}[-i]\right) \\
& =\chi\left(A^{\bullet}\right)
\end{aligned}
$$

where the second equality follows from the commutativity of (4), the third from the defining relations of $K_{1}\left(\Lambda(G)_{S^{*}}\right)$ and the fourth from (6). This completes our proof of Theorem 4.1(ii). 
Lemma 4.4. Let $P^{\bullet}$ in $C^{\mathrm{p}}(\Lambda(G))$ be as in (4) and assume that $G$ has rank one. If $\alpha: P^{\bullet} \rightarrow P^{\bullet}$ is any morphism of complexes for which $H^{i}(\alpha)$ is injective in each degree $i$, then there is a morphism of complexes $\hat{\alpha}: P^{\bullet} \rightarrow P^{\bullet}$ that is homotopic to $\alpha$ and injective in each degree.

Proof. In each degree $i$ there are tautological exact sequences

$$
\begin{aligned}
& 0 \longrightarrow Z^{i} \longrightarrow P^{i} \stackrel{d^{i}}{\longrightarrow} B^{i+1} \longrightarrow 0 \\
& 0 \longrightarrow B^{i} \longrightarrow Z^{i} \longrightarrow H^{i} \longrightarrow 0
\end{aligned}
$$

where $B^{i}, Z^{i}$ and $H^{i}$ are the coboundaries, cocycles and cohomology of $P^{\bullet}$.

Now every finitely generated $\Lambda(G)\left[\frac{1}{p}\right]$-module is of projective dimension at most one. (Indeed, by [7, Prop. (5.3.19)(i)], this claim is true if we replace $G$ by any (open) subgroup that is topologically isomorphic to $\mathbb{Z}_{p}$ and then the result for $G$ itself follows by the argument of [6, Prop. 4.3.4].) Thus, the (image under $\mathbb{Q}_{p} \otimes_{\mathbb{Z}_{p}}$ - of the) exact sequences (7) allow one to prove, by descending induction on $i$, that each $\Lambda(G)[1 / p]$-module $\mathbb{Q}_{p} \otimes_{\mathbb{Z}_{p}} B^{i}$ is projective. The upper sequence of (7) therefore splits after applying $\mathbb{Q}_{p} \otimes_{\mathbb{Z}_{p}}-$ and so in each degree $i$ we can choose a $\Lambda(G)$-submodule $\hat{B}^{i+1}$ of $P^{i}$ such that $\hat{B}^{i+1} \cap Z^{i}=0$ and $B^{i+1} / d^{i}\left(\hat{B}^{i+1}\right)$ is $p$-torsion.

We next construct a homomorphism of $\Lambda(G)$-modules $k^{i}: P^{i} \rightarrow \hat{B}^{i}$ with

$$
k^{i}\left(\hat{B}^{i+1}\right)=0
$$

and such that the quotient module

$$
B^{i} /\left(d^{i-1} \circ\left(\alpha^{i-1}+k^{i} \circ d^{i-1}\right)\left(\hat{B}^{i}\right)\right)
$$

is $p$-torsion. To do this we claim first that the cokernel of the map

$$
\eta^{i}: \operatorname{Hom}_{\Lambda(G)}\left(P^{i} / \hat{B}^{i+1}, \hat{B}^{i}\right) \rightarrow \operatorname{Hom}_{\Lambda(G)}\left(B^{i}, \hat{B}^{i}\right)
$$

that is induced by the composite $B^{i} \subset P^{i} \rightarrow P^{i} / \hat{B}^{i+1}$ is $p$-torsion. Indeed, the natural map $Z^{i} \rightarrow P^{i} / \hat{B}^{i+1}$ is injective with $p$-torsion cokernel and so there is a natural complex $\operatorname{Hom}_{\Lambda(G)}\left(P^{i} / \hat{B}^{i+1}, \hat{B}^{i}\right) \rightarrow \operatorname{Hom}_{\Lambda(G)}\left(B^{i}, \hat{B}^{i}\right) \rightarrow$ $\operatorname{Ext}_{\Lambda(G)}^{1}\left(H^{i}\left(P^{\bullet}\right), \hat{B}^{i}\right)$ which has $p$-torsion cohomology in the central degree. Thus one has $\mathbb{Q}_{p} \otimes_{\mathbb{Z}_{p}} \operatorname{cok}\left(\eta^{i}\right)=0$ if $\mathbb{Q}_{p} \otimes_{\mathbb{Z}_{p}} \operatorname{Ext}_{\Lambda(G)}^{1}\left(H^{i}\left(P^{\bullet}\right), \hat{B}^{i}\right)=0$. But $\mathbb{Q}_{p} \otimes_{\mathbb{Z}_{p}}$ $\hat{B}^{i}$ is a projective $\Lambda(G)[1 / p]$-module and so $\mathbb{Q}_{p} \otimes_{\mathbb{Z}_{p}} \operatorname{Ext}_{\Lambda(G)}^{1}\left(H^{i}\left(P^{\bullet}\right), \hat{B}^{i}\right)=$ 0 if $\mathbb{Q}_{p} \otimes_{\mathbb{Z}_{p}} \operatorname{Ext}_{\Lambda(G)}^{1}\left(H^{i}\left(P^{\bullet}\right), \Lambda(G)\right)=0$. Moreover, the module $H^{i}\left(P^{\bullet}\right)$ is isomorphic to $\Lambda(G) \otimes_{\Lambda(H)} H^{i}\left(A^{\bullet}\right)$ and so $\operatorname{Ext}_{\Lambda(G)}^{1}\left(H^{i}\left(P^{\bullet}\right), \Lambda(G)\right)$ is isomorphic to $\operatorname{Ext}_{\Lambda(H)}^{1}\left(H^{i}\left(A^{\bullet}\right), \Lambda(H)\right) \otimes_{\Lambda(H)} \Lambda(G)$ (cf. the discussion following [8, 
Prop. 3.1]). To prove that $\operatorname{cok}\left(\eta^{i}\right)$ is $p$-torsion it is thus enough to note that $\mathbb{Q}_{p} \otimes_{\mathbb{Z}_{p}} \operatorname{Ext}_{\Lambda(H)}^{1}\left(H^{i}\left(A^{\bullet}\right), \Lambda(H)\right)=\mathbb{Q}_{p} \otimes_{\mathbb{Z}_{p}} \operatorname{Ext}_{\mathbb{Z}_{p}[H]}^{1}\left(H^{i}\left(A^{\bullet}\right), \mathbb{Z}_{p}[H]\right)$ vanishes because $H$ is finite.

Next we note that the vanishing of $\mathbb{Q}_{p} \otimes_{\mathbb{Z}_{p}}\left(B^{i+1} / d^{i}\left(\hat{B}^{i+1}\right)\right)$ implies that the kernel and cokernel of the homomorphism $\operatorname{Hom}_{\Lambda(G)}\left(B^{i}, \hat{B}^{i}\right) \rightarrow \operatorname{Hom}_{\Lambda(G)}\left(\hat{B}^{i}\right.$, $\left.B^{i}\right)$ that sends $k$ to $d^{i-1} \circ k \circ d^{i-1}$ are both $p$-torsion. Since $\mathbb{Q}_{p} \otimes_{\mathbb{Z}_{p}} \operatorname{cok}\left(\eta^{a}\right)=0$ in each degree $a$ this allows us to choose $k^{i}$ in $\operatorname{Hom}_{\Lambda(G)}\left(P^{i} / \hat{B}^{i+1}, \hat{B}^{i}\right)$ so that $d^{i-1} \circ \alpha^{i}+d^{i-1} \circ k^{i} \circ d^{i-1} \in \operatorname{Hom}_{\Lambda(G)}\left(\hat{B}^{i}, B^{i}\right)$ has $p$-torsion kernel and $p$ torsion cokernel. Then these homomorphisms $k^{i}$ satisfy both (8) and (9) and we set $\hat{\alpha}^{i}:=\alpha^{i}-\left(d^{i-1} \circ k^{i}+k^{i+1} \circ d^{i}\right)$. We thereby obtain a morphism of complexes $\hat{\alpha}$ that is homotopic to $\alpha$ via the homotopy $\left\{-k^{i}\right\}_{i \in \mathbb{Z}}$. Also (8) and (9) combine to imply that $B^{i+1} / d^{i}\left(\hat{\alpha}^{i}\left(\hat{B}^{i+1}\right)\right)$ is $p$-torsion in each degree $i$. But each map $d^{i}: \hat{B}^{i+1} \rightarrow B^{i}$ is injective with $p$-torsion cokernel and hence $\hat{\alpha}^{i+1}: B^{i+1} \rightarrow B^{i+1}$ is also injective with $p$-torsion cokernel in each degree $i+1$. In addition $H^{i}(\hat{\alpha})=H^{i}(\alpha)$ is assumed to be injective in each degree $i$ and hence, by an easy exercise involving the exact sequences (7), we may deduce that each homomorphism $\hat{\alpha}^{i}$ is itself injective, as required.

\section{References}

[1] M. Breuning and D. Burns, Additivity of Euler characteristics in relative algebraic $K$ groups, Homology Homotopy Appl. 7 (2005), no. 3, 11-36 (electronic).

[2] D. Burns and M. Flach, Tamagawa numbers for motives with (non-commutative) coefficients, Doc. Math. 6 (2001), 501-570 (electronic).

[3] D. Burns and O. Venjakob, On descent theory and main conjectures in non-commutative Iwasawa theory, to appear in J. Inst. Math. Jussieu.

[4] J. Coates, T. Fukaya, K. Kato, R. Sujatha and O. Venjakob, The $\mathrm{GL}_{2}$ main conjecture for elliptic curves without complex multiplication, Publ. Math. Inst. Hautes Études Sci. No. 101 (2005), 163-208.

[5] J. Coates, P. Schneider and R. Sujatha, Links between cyclotomic and $\mathrm{GL}_{2}$ Iwasawa theory, Doc. Math. 2003, Extra Vol., 187-215 (electronic).

[6] T. Fukaya and K. Kato, A formulation of conjectures on $p$-adic zeta functions in noncommutative Iwasawa theory, in Proceedings of the St. Petersburg Mathematical Society. Vol. XII, 1-85, Amer. Math. Soc. Transl. Ser. 2, 219, Amer. Math. Soc., Providence, RI, 2006.

[7] J. Neukirch, A. Schmidt and K. Wingberg, Cohomology of number fields, Springer, Berlin, 2000.

[8] P. Schneider and O. Venjakob, On the codimension of modules over skew power series rings with applications to Iwasawa algebras, J. Pure Appl. Algebra 204 (2006), no. 2, 349-367.

[9] R. G. Swan, Algebraic K-theory, Lecture Notes in Math., 76, Springer, Berlin, 1968.

[10] O. Venjakob, Characteristic elements in noncommutative Iwasawa theory, J. Reine Angew. Math. 583 (2005), 193-236. 